\title{
溶液中络合物逐级稳定常数与配位数之间的 $\mathbf{S}$ 型关系和直线关系
}

\author{
张 正 斌 \\ (山东海洋学院海洋化学系)
}

溶液中络合物逐级稳定常数与配位数之 间的定量关系问题已有不少人作了研究 ${ }^{[1-5]}$. 但是 Bjerrum 的计算结果与实验结果相差较 $大^{[1,3]}$. Бобко ${ }^{[4]}$ 和马登勇 ${ }^{[3]}$ 是在 Хадаков 计 算酸解离常数公式的基础上讨论这个问题 的,鉴于 Хадаков 公式是十分粗略的, 仅考虑 了静电效应而与稳定常数直接联系起来 (相 当于 $\Delta H=-R T \ln K)$, 故此法值得研究. 目前最流行的是 Panthaleon ${ }^{[2]}$ 对一些体系研 究后提出的直线关系, 即 $\log K_{n}=\log K_{1}$ -

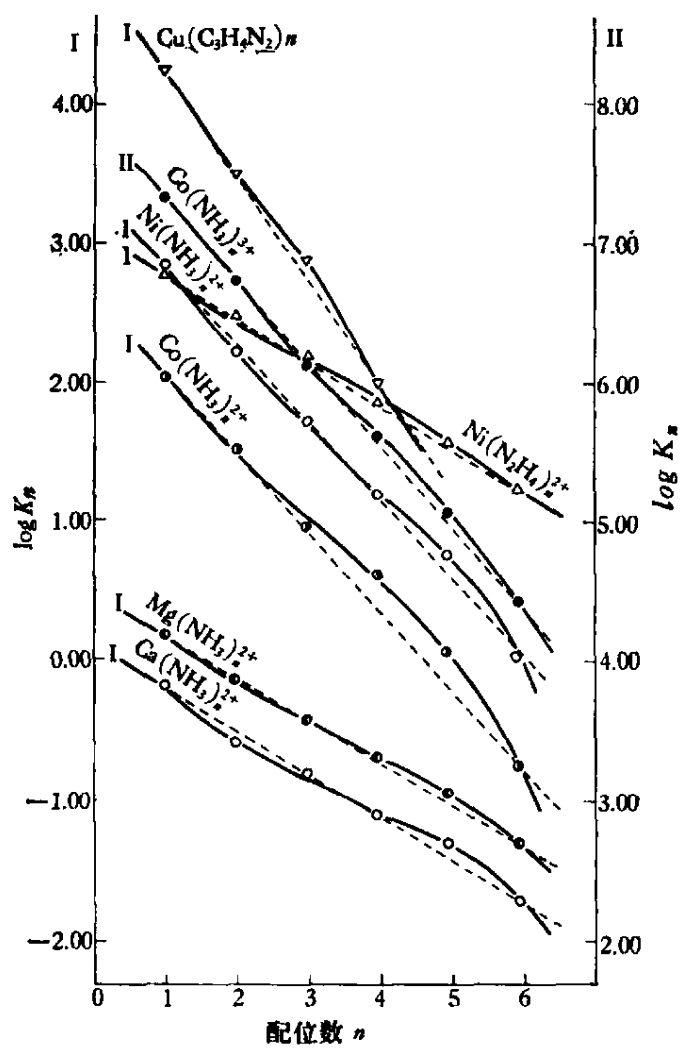

图 1 一些氨络合物的 $\log K_{n}$ 与 $n$ 的 $S$ 型关系 $(n>3)$
$2 \lambda(n-1)$. 二十年来, 不少人对此式在理论 上 $^{[3,5]}$ 和实验上作了解释和论证。我们研究了 几百个络合物体系 ${ }^{[6]}$ (包括前人 ${ }^{[2,3]}$ 认为典型 的直线关系的体系)，结果发现：(1) 只有当 最大配位数 $N \leqslant 3$ 时, $\log K_{n}$ 与 $n$ 才呈直线 关系.（2）在 $N>3$ 的绝大多数情况下(例 如 $N=4,5,6$ 等), $\log K_{n}$ 与 $n$ 不存在直线 关系, 而微呈 “ $\mathrm{S}$ ” 型关系. 结果如图 1-6 所 示.

根据热力学公式:

$\Delta G_{n}=-R T \ln K_{n}^{0}=\Delta H_{n}-T \Delta S_{n}$ ，(1)

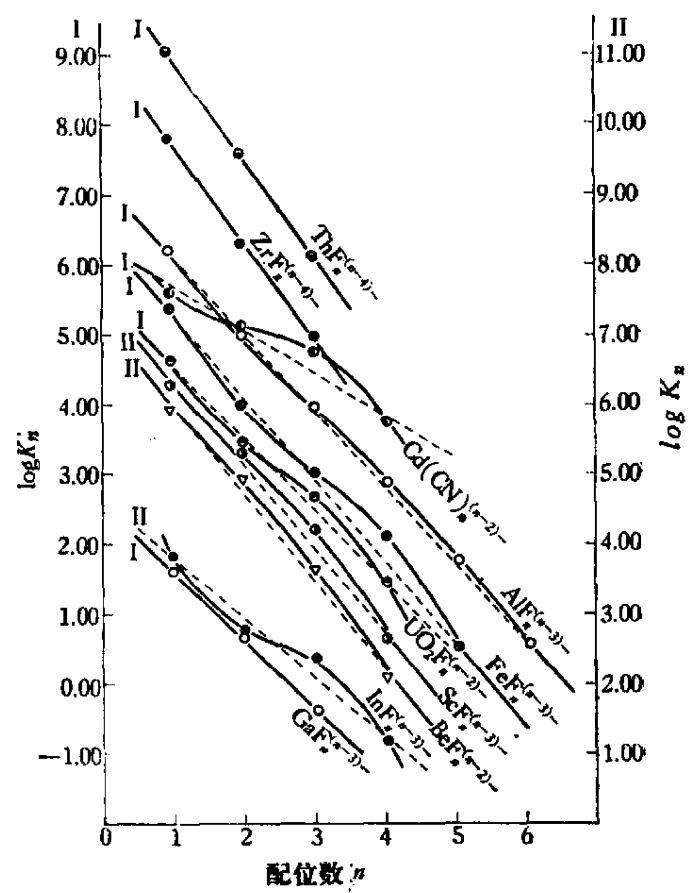

图 2 一些偂的络合物的 $\log K_{n}$ 与 $n$ 的 $S$ 型 关系 $(n>3)$ 及直线关系 $(n \leqslant 3)$

本文 1973 年 5 月 21 日收到. 


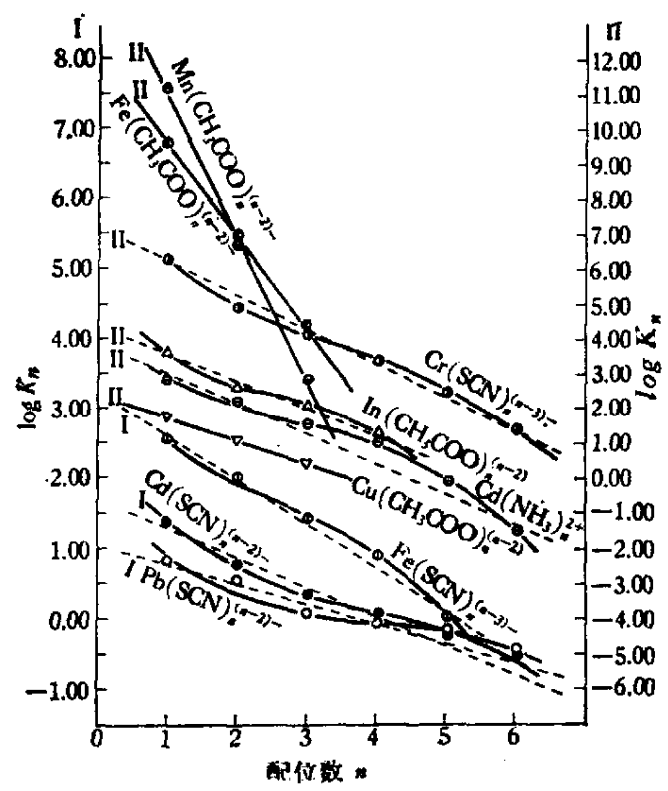

图 3 一些硫氢酸及醋酸等的络合物的 $\log K_{n}$ 与 $n$ 的 $S$ 型关系 $(n>3)$ 和直线关系 $(n \leqslant 3)$

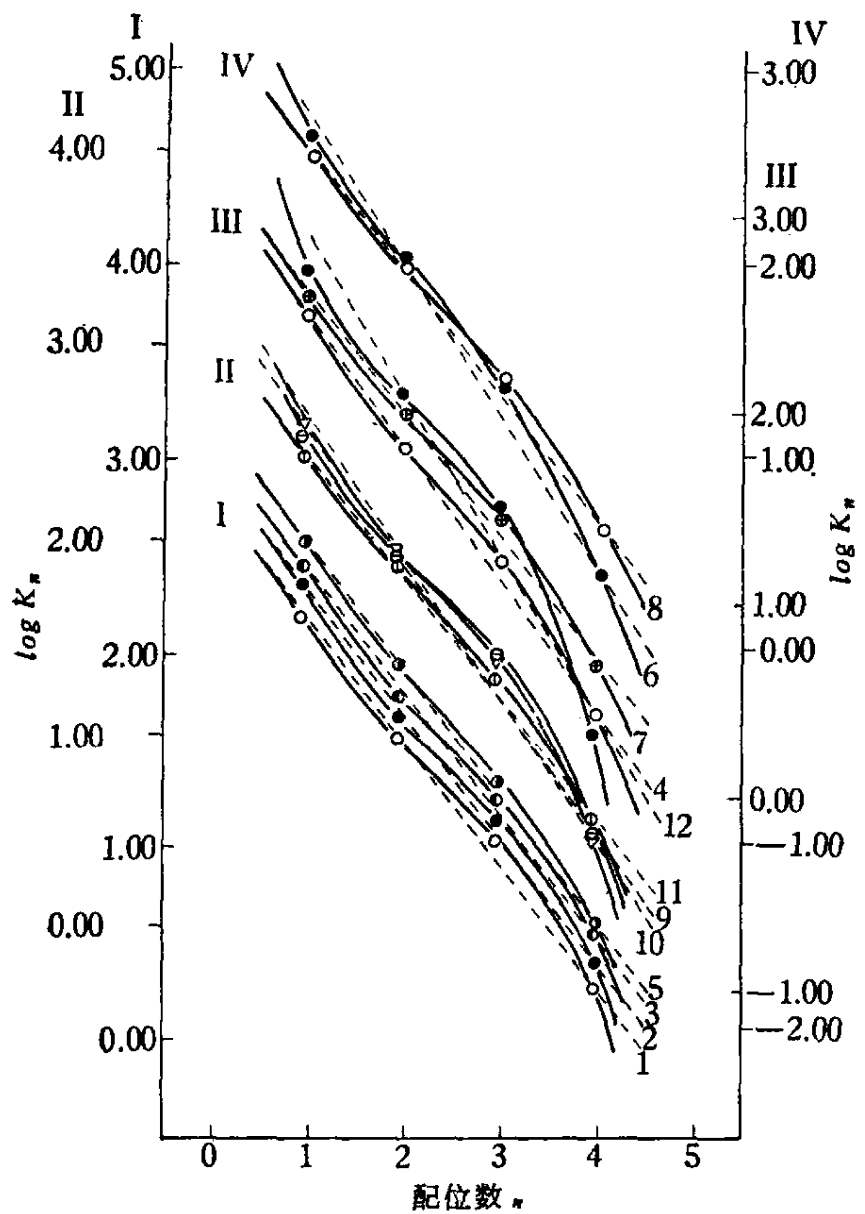

图 4 一些乙醇酸络合物的 $\log K_{n}$ 与 $n$ 的 $\mathrm{S}$ 型关系 1. $\mathrm{La}\left(\mathrm{HOCH}_{2} \mathrm{COO}\right)_{n}^{(n-3)-}, 2 . \mathrm{Ce}\left(\mathrm{HOCH}_{2} \mathrm{COO}\right)_{n}^{(n-3)-}$, 3. $\operatorname{Pr}\left(\mathrm{HOCH}_{2} \mathrm{COO}\right)_{n}^{(n-3)-}$, 4. $\mathrm{Nd}\left(\mathrm{HOCH}_{2} \mathrm{COO}\right)^{(n-3)-}$, 5. $\mathrm{Sm}\left(\mathrm{HOCH}_{2} \mathrm{COO}\right)_{n}^{(n-3)-}$, 6. $\mathrm{Eu}\left(\mathrm{HOCH}_{2} \mathrm{COO}\right)^{(n-3)-}$, 7. $\mathrm{Gd}\left(\mathrm{HOCH}_{2} \mathrm{COO}\right)_{n}^{(n-3)-}$, 8. $\mathrm{Dy}\left(\mathrm{HOCH}_{2} \mathrm{COO}\right)_{n}^{(n-3)-}$, 9. $\mathrm{Ho}\left(\mathrm{HOCH}_{2} \mathrm{COO}\right)_{n}^{(n-3)-}, 10 . \mathrm{Er}\left(\mathrm{HOCH}_{2} \mathrm{COO}\right)_{n}^{(n-3)-}$, 11. $\mathrm{Y}\left(\mathrm{HOCH}_{2} \mathrm{COO}\right)_{n}^{(n-3)-}$, 12. $\mathrm{Yb}\left(\mathrm{HOCH}_{2} \mathrm{COO}\right)_{n}^{(n-3)-}$.

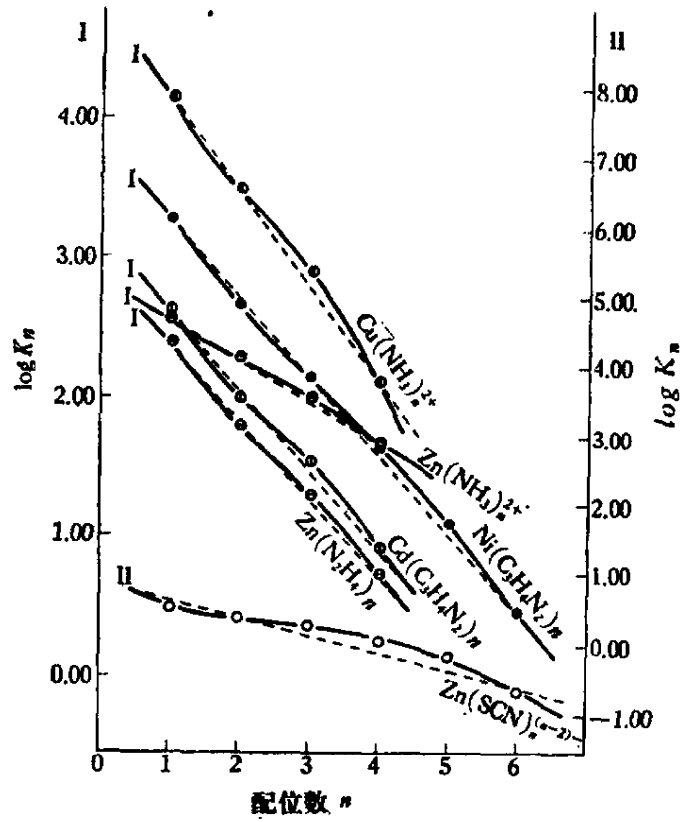

图 5 一些络合物的 $\log _{n}$ 与 $n$ 的 $\mathrm{S}$ 型关系

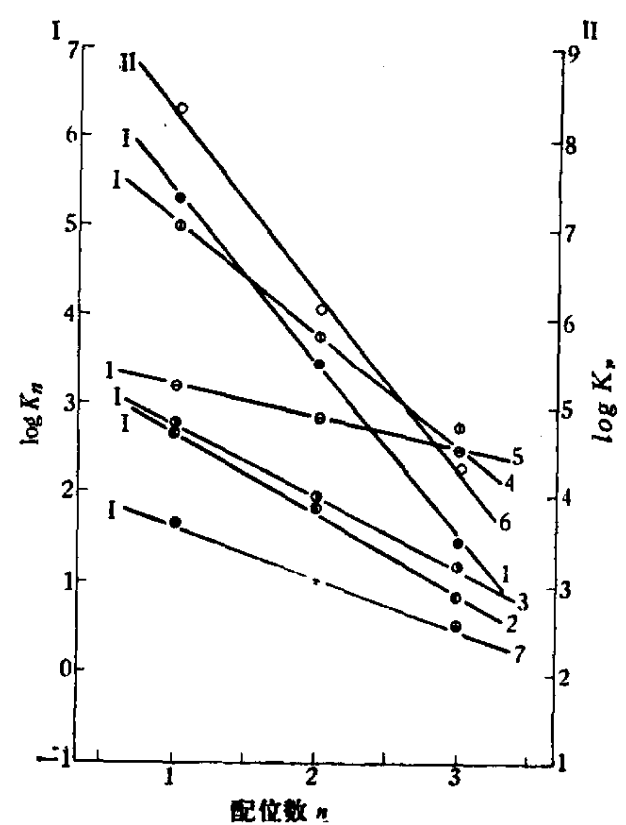

图 6 当 $n=3$ 时, 钝 $\left(\mathrm{Ce}^{3+}\right)$ 的一些络合物的 $\log K_{n}$ 与 $n$ 的直线关系
1. $\mathrm{C}_{2} \mathrm{H}_{2} \mathrm{O}_{4}$,
2. $\mathrm{C}_{2} \mathrm{H}_{4} \mathrm{O}_{3}$,
3. $\mathrm{C}_{3} \mathrm{H}_{6} \mathrm{O}_{3}$,
4. $\mathrm{C}_{4} \mathrm{H}_{6} \mathrm{O}_{3}$,
5. $\mathrm{C}_{4} \mathrm{H}_{7} \mathrm{O}_{4} \mathrm{~N}$
6. $\mathrm{C}_{7} \mathrm{H}_{5} \mathrm{O}_{4} \mathrm{~N}$.
7. $\mathrm{C}_{2} \mathrm{H}_{4} \mathrm{O}_{2}$. 
$\log K_{n}^{0} 、 \log K_{1}^{0}$ 和 $n$ 的关系主要决定于静电 效应、统计效应和溶剂化效应. $\Delta S_{n}$ 根据 Bjerrum $^{[1]}$ 的统计效应为:

$$
\Delta S_{n}-\Delta S_{1}=R \ln \frac{N-n+1}{n N},
$$

静电效应因前人的计算方法比较粗粮，我们

$$
\begin{gathered}
\Delta H_{M L_{n}}^{(s)}=-\left\{\frac{Z_{M} \sum_{i} C_{i-} Z_{i-}}{\sum_{i} C_{i-}}+\frac{n Z_{L} \sum_{j} C_{i+} Z_{j+}}{\sum_{i} C_{j+}}\right\} \frac{N^{1 / 3} e^{2} M_{n} f\left(\frac{298}{T}\right)^{1 / 2} C_{\mathrm{B}}^{1 / 3}}{10 D}+B_{M L_{n},} \\
\Delta H_{M L_{n-1}}^{(s)}=-\left\{\frac{Z_{M} \sum_{i} C_{i-} Z_{i-}}{\sum_{i} C_{i-}}+\frac{(n-1) Z_{L} \sum_{i} C_{i+} Z_{i+}}{\sum_{i} C_{i+}}\right\} \frac{N^{1 / 3} e M_{n} f\left(\frac{298}{T}\right)^{1 / 2} C_{8}^{1 / 3}}{10 D}+B_{M L_{n-1}},
\end{gathered}
$$

式中符号的意义与文献 [8] 相似. $\Delta H_{n}$ 主要 包括静电效应和溶剂化效应。如只考虑静电 效应和统计效应, 则由式 (1) 得:

$$
\begin{aligned}
\Delta G_{n} & =\Delta G_{M L_{n}}-\Delta G_{M L_{n-1}} \\
& =\left[\Delta H_{M L_{n}}^{(s)}-\Delta H_{M L_{n-1}}^{(s)}\right]-T \Delta S_{n} \\
& =-R T \ln \frac{\beta_{n}}{\beta_{n-1}}=-R T \ln K_{n}^{0} \\
& =-2.303 R T \log K_{n 。}^{0} .
\end{aligned}
$$

建议用 Frank 和 Thompson ${ }^{[8]}$ 的方法计算. 因为对络合物溶液体系比一般电解质更符合 “近程有序而远程无序”的假定. 用此计算法 于络合物体系，对络离子 $M L_{n}$ 和 $M L_{n-1}$ 得:

$$
\log K_{n}^{0}=\left\{\frac{Z_{L} \sum_{j} C_{i+} Z_{i+}}{\sum_{i} C_{i+}}\right\} \frac{N^{1 / 3} e^{2} f\left(\frac{298}{T}\right)^{1 / 2} C_{8}^{1 / 3}}{2.303 R T 10 D}(2 n-1) M_{1}+\frac{T \Delta S_{n}}{2.303 R T},
$$

用类似方法可得:

$$
\log K_{1}^{0}=\left\{\frac{Z_{L} \sum_{i} C_{i+} Z_{i+}}{\sum_{i} C_{i+}}\right\} \frac{N^{1 / 3} e^{2} f\left(\frac{298}{T}\right)^{1 / 2} C_{\mathrm{B}}^{1 / 3}}{2.303 R T 10 D} M_{1}+\frac{T \Delta S_{1}}{2.303 R T} .
$$

由式 (5)、(6) 和 (2) (注意 $Z_{L}$ 是个负数, 如下述式中只取其绝对值)，得:

$$
\log K_{n}^{0}=\log K_{1}^{0}-\left\{\frac{Z_{L} \sum_{i} C_{i+} Z_{i+}}{\sum_{i} C_{i+}}\right\} \frac{N^{1 / 3} e^{2} f\left(\frac{298}{T}\right)^{1 / 2} C_{8}^{1 / 3}}{2.303 R T 10 D} 2 M_{1}(n-1)+\log \frac{N-n+1}{n N} .
$$

根据 Davies 方程式 ${ }^{[9-11]}$, 热力学稳定常数 $K_{n}^{0}$ 与表观稳定常数 $K_{n}$ 之间关系式为:

$$
\log K_{n}=\log K_{n}^{0}-\Delta Z^{2} A\left(\frac{\mu^{1 / 2}}{1+B \mu^{1 / 2}}-C \mu\right) \text {, }
$$

在 $\mu$ 恒定时，得：

$$
\log K_{n}-\log K_{1}=\log K_{n}^{0}-\log K_{1}
$$

并注意到 $l=\left(\frac{1000}{N C_{\text {量 }}}\right)^{1 / 3}$, 再将 $N, R, e, D$ （对水为 78.5) 的具体数值代入式 (7), 则得: 


$$
\log K_{n}=\log K_{1}-\log \frac{N n}{N-n+1}-\left\{\frac{Z_{L} \sum_{j} C_{i+} Z_{i+}}{\sum_{j} C_{i+}}\right\} \frac{3.1 f\left(\frac{298}{T}\right)^{1 / 2}}{l} 2 M_{1}(n-1) .
$$

如果进一步考虑溶剂化作用(水化作用)，静电作用可用上述原理和方法计算，则最后 并假定偶极水分子与中心离子 $M^{z+}$ 之间的 得:

$$
\begin{aligned}
\log K_{n}= & \log K_{1}-\log \frac{N n}{N-n+1}-\left\{\left[\frac{Z_{L} \sum_{j} C_{i+} Z_{i+}}{\sum_{i} C_{i+}}\right] \frac{3.1 f\left(\frac{298}{T}\right)^{1 / 2}}{l} 2 M_{1}\right. \\
& \left.-\left[\frac{\left(\mu_{0}+\mu_{i}\right)_{\left(\mathrm{H}_{2} \mathrm{O}\right)}^{2}}{r_{M-\mathrm{H}_{2} \mathrm{O}}^{2}} \frac{\sum_{j} C_{i+} Z_{j+}}{\sum_{i} C_{j+}}\right] \frac{3.1 f_{\left(\mathrm{H}_{2} \mathrm{O}\right)}^{\prime}\left(\frac{298}{T}\right)^{1 / 2} M_{1\left(\mathrm{H}_{2} \mathrm{O}\right)}^{\prime}}{r_{M-\mathrm{H}_{2} \mathrm{O}}}\right\}(n-1),
\end{aligned}
$$

式中 $\mu_{0}$ 和 $\mu_{i}$ 分别是永久偶极矩和诱导偶极 位体 (如 $\mathrm{NH}_{3}$ 等), 用同样原理可得:

矩. 如果配位体不是离子, 而是中性分子配

$$
\begin{aligned}
\log K_{n}= & \log K_{1}-\log \frac{N n}{N-n+1}-\left\{\left[\frac{\left(\mu_{0}+\mu_{i}\right)_{L}^{2}}{r_{M-L}^{2}} \frac{\sum_{i} C_{i+} Z_{i+}}{\sum_{i} C_{i+}}\right] \frac{3.1 M_{1(L)}^{\prime} f_{(L)}^{\prime}\left(\frac{298}{T}\right)^{1 / 2}}{r_{M-L}}\right. \\
& \left.-\left[\frac{\left(\mu_{0}-\mu_{i}\right)_{\mathrm{H}_{2} \mathrm{O}}^{2}}{r_{M-\mathrm{H}_{2} \mathrm{O}}^{2}} \frac{\sum_{i} C_{i+} Z_{i+}}{\sum_{i} C_{i+}}\right] \frac{3.1 M_{1\left(\mathrm{H}_{2} \mathrm{O}\right) f_{\left(\mathrm{H}_{2} \mathrm{O}\right)}^{\prime \prime}\left(\frac{298}{T}\right)^{1 / 2}}^{r_{M-\mathrm{H}_{2} \mathrm{O}}}}{r^{\prime 2}}\right](n-1),
\end{aligned}
$$

式中 $r_{M-L}$ 和 $r_{M-\mathrm{H}_{2}} \mathrm{O}$ 分别是 $M$ 与 $L$ 和 $\mathrm{H}_{2} \mathrm{O}$ 间 距离, $M_{1(L)}^{\prime}$ 和 $M_{1\left(\mathrm{H}_{2} \mathrm{O}\right)}^{\prime \prime}$ 的意义同 $M_{1}$, 仅其值 随 $\mathrm{H}_{2} \mathrm{O}$ 或 $L$ 之不同而异.

式 (9) 和 (10) 是本文推导得出的一般方 程式. 计算起来并不复杂, 因 $K_{1}, N, Z_{L}, C_{j+}$, $Z_{i+}, l$ (溶液中离子的平均距离) $, \mu_{0}, \mu_{i}$, 或 $\alpha, r_{M-L}$ 和 $r_{M-\mathrm{H}_{2} \mathrm{O}}$ 等值, 均可从手册中查到. 仅 $M_{1} f\left(\frac{298}{T}\right)^{1 / 2}$ 不易查到. 根据文献 [8]中公 式: $M_{1} f\left(\frac{298}{T}\right)^{1 / 2}=\frac{v}{\sum_{i}\left(\nu_{i} Z_{i}\right)^{2}} \frac{3 d}{e^{2} / 2 D k T}$ 可 得:

$$
\frac{\left\{M_{1} f\left(\frac{298}{T}\right)^{1 / 2}\right\}_{a}}{\left\{M_{1} f\left(\frac{298}{T}\right)^{1 / 2}\right\}_{\beta}}=\frac{\left\{\frac{v}{\sum_{i}\left(\nu_{i} Z_{i}\right)^{2}}\right\}_{\alpha}}{\left\{\frac{\nu}{\sum_{i}\left(\nu_{i} Z_{i}\right)^{2}}\right\}_{\beta}},
$$

由已知络离子 $\alpha$ 的 $\left\{M_{1} f\left(\frac{298}{T}\right)^{1 / 2}\right\}_{\alpha}$ 值, 可求 另一络离子 $\beta$ 的末知的 $\left\{M_{1} f\left(\frac{298}{T}\right)^{1 / 2}\right\}_{\beta}$ 值. $M_{1(L)}^{\prime} f_{(L)}^{\prime}\left(\frac{298}{T}\right)^{1 / 2}$ 和 $M_{1\left(\mathrm{H}_{2} \mathrm{O}\right)}^{\prime \prime} f_{\left(\mathrm{H}_{2} \mathrm{O}\right)}^{\prime \prime}\left(\frac{298}{T}\right)^{1 / 2}$ 可用 类似方法求得. 另外 $M_{1} f\left(\frac{298}{T}\right)^{1 / 2}$ 也可在 $K_{n}, K_{1}, N, n, Z_{L}, Z_{i}, C_{i+}, l$ 等值已知后用 式(9)计算而得. $M_{1(L)}^{\prime} f_{(L)}^{\prime}\left(\frac{298}{T}\right)^{1 / 2}$ 和 $M_{1\left(\mathrm{H}_{2} \mathrm{O}\right)}^{\prime \prime}$ $f_{\left(\mathrm{H}_{2} \mathrm{O}\right)}^{\prime \prime}\left(\frac{298}{T}\right)^{1 / 2}$ 可用类似方法由式(10)和 (11) 计算而得, 然后以一络离子之值为基准, 用与 式(12)类似的公式求另一络离子的值. 在目 前 $M_{1(L)}^{\prime} f_{(L)}^{\prime}\left(\frac{298}{T}\right)^{1 / 2}$ 和 $M_{1\left(\mathrm{H}_{2} \mathrm{O}\right)}^{\prime \prime} f_{\left(\mathrm{H}_{2} \mathrm{O}\right)}^{\prime \prime}\left(\frac{298}{T}\right)^{1 / 2}$ 值 较贫乏的情况下, 后一方法是可行的, 至少可 作校核数值用. 
根据式 (10) 和 (11) 计算得的结果如图 1-6 中实线所示. 此外, 运用上述观点我们 还研究了几百个络合物体系 ${ }^{[6]}$, 结果表明: (1) $\log K_{n}$ 与 $n$ 呈直线关系的看法, 仅在 $N \leqslant 3$ 时正确 (图 2、3 和 6 仅是大量例中之一斑). 在 $N>3$ 时, 即使是前人典型的直线关系体 系也是与事实不符的, 应呈 “ $\mathrm{s}$ ” 型曲线关系。 如此之多的体系无例外地有共同的 “ $S$ ” 型规 律 (即与直线关系相比, $\log K_{2}$ 一律 偏 低, $\log K_{4}, \log K_{5}$ 一律偏高), 这是以统计性为特 点的“误差论”所不能解释的. (2) 公式 (10) 和 (11) 能定性和定量地说明实验事实. 静电 效应和溶剂化效应所属的式中右边第 3 项, 是决定 $\log K_{n}$ 与 $n$ 呈直线关系的, 这是文 献 $[2,3]$ 等认为呈直线关系的根据. 但统计效 应所属的 $\log \frac{N n}{N-n+1}$ 与 $n$ 的关系, 在 $N>3$ 时呈反 “S” 型(参看图 7), 仅在 $N \leqslant 3$

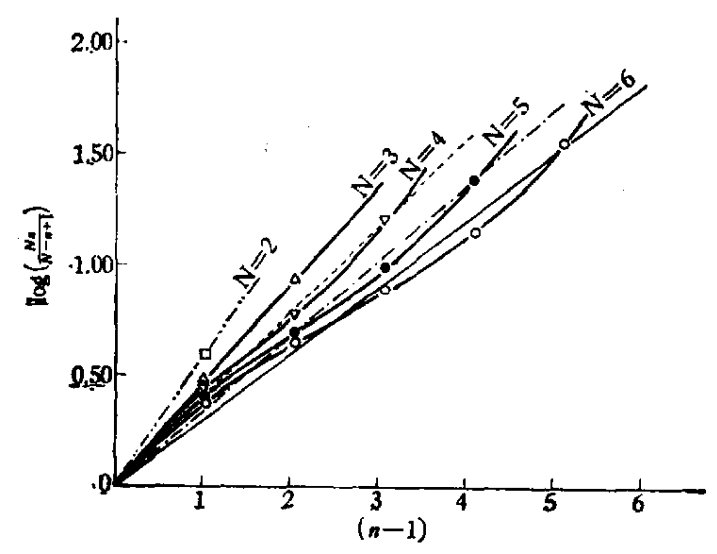

图 $7 \log \frac{N n}{N-n+1}$ 与 $(n-1)$ 的反 $s$ 型关系 $(n>3)$ 和直线关系 $(n \leqslant 3)$

时呈直线关系. 这就使 $\log K_{n}$ 与 $n$ 的关系 在 $N=4,5, \cdots$ 时微呈 “ $\mathrm{S}$ ” 型关系, 而 $N \leqslant 3$ 时呈直线关系. “ $\mathrm{S}$ ” 型是否显著, 与 $\log \frac{N n}{N-n+1}$ 值的贡献大小有关,一般贡献 并不大, 致使文献 $[2,3]$ 等误认为直线关系. (3)在几百个络合物体系中, 只有几十个体系
$N \geqslant 4^{[6]}$, 适用于本文来研究 $\log K_{n}$ 与 $n$ 的关 系. 它们又分成两类, 一类是 $\log K_{n}$ 与 $n$ 呈 “ $s$ ”型关系 $(N>3$ 时)或直线关系 $(N \leqslant 3$ 时), 这些体系主要是氨化物、攴化物 (主要是氟化 物)、硫氯化物、胼合物和 $\mathrm{CH}_{3} \mathrm{COO}^{-} 、 \mathrm{C}_{3} \mathrm{H}_{4} \mathrm{~N}_{2}$ 等的络合物. 另一类是 $\log K_{n}$ 与 $n$ 呈非线关 系(不是 $\mathrm{S}$ 型关系)。本文主要论证前一类络 合物体系, 并非如前人所述是直线型的, 而是 $N>3$ 呈 “ $\mathrm{S}$ ” 型曲线关系, $N \leqslant 3$ 时呈直线 关系. 此结论主要适用于电价络合物体系. 而用本文理论对后一类络合物的研究，因与 络合物构型、键性质、结构等因素有关，比较 复杂,将另文报道.

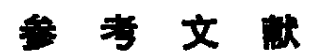

[1] Bjerrum, J., "Metal Ammine Formation in Aqueous Solution", Haase P. \& Son, Copenhagen, 1941; Sen, B., Anal. Chim. Acta., 27 (1962), 515.

[2] van Panthaleon, C. L., van Eck, Rec. trav. chim., 72 (1953), 529.

[ 3 ] 马登勇, 化学通报, 1964, [4], 208, 及文末所 引有关文献。

[4] Бабко, А. К., «Физико-химический анализ цомплексдых соединений в растворах》. Кнев, 1955.

[ 5 ] Фридман, Я. Д., Ж. неорг, хим., 4 (1961), 1501.

[6] Sillen, L. G., ed., Stability Constants of Metal-Ion Complexes, The Chem. Soc., London, 1964.

[ 7 ] Moeller, T., Martin, D. F., Thompson, L. C., Ferrús, R., Feistel, G. R. and Randall, W. J., Chem. Rev., 65 (1965), 1.

[8] Frank, H. S. and Thompson, P. T., "A. Point of View on Ion Clouds" in Hamer, W. J., "The Structure of Electrolytic Solution", John Wiley \& Sons, Inc., New York, 1959, 113.

[9] Rossotti, F. J. C. and Rossotti, H., "The Determination of Stability Constant", McGraw-Hill, New York, 1961, 30-34.

[10] Яцимирский, К. Б., ЖЖ. неорг. хим., 2 (1957), 293.

[11] Васильв, В. П. в др., Ж. неорг. хим., 7 (1962), 1788; 8 (1963), 1895; 9 (1964), 467. 